\title{
Effect of Core Training on Trunk Flexor Musculature in Male Soccer Players
}

\section{(ㄷ) (2) (1) (-)}

\author{
Authors

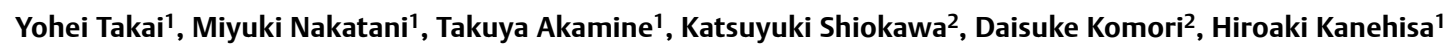

\author{
Affiliations \\ 1 National Institute of Fitness and Sports in Kanoya, Sports \\ and Life sciences, Kanoya, Japan \\ 2 National Institute of Fitness and Sports in Kanoya, Sports \\ and Budo Practice, Kanoya, Japan
}

\author{
Key words \\ dual-energy X-ray absorptiometry, ultrasound, stabilization, \\ a wheeled platform, body mass-based exercise \\ received 11.04 .2017 \\ revised 28.05.2017 \\ accepted 22.06.2017

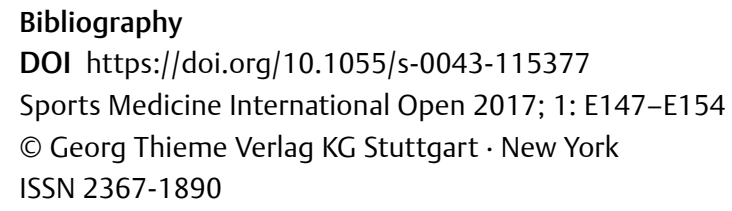

\begin{abstract}
The present study aimed to elucidate the effect of core training on trunk flexor musculature in athletes. Twenty-eight collegiate male soccer players were randomly assigned to three groups: a training group that performed core exercises with wheeled platforms (WP), a training group that performed body massbased core exercise (BME), and a control group that did not perform core exercise training (CON). WP and BME trained twice a week for 10 weeks. The WP performed 8-14 exercises with wheeled platforms. BME conducted four core exercises to failure. Before and after the intervention, trunk segment lean body mass (LBM) was measured using a whole-body dual-energy X-ray absorptiometry scanner. Muscle thicknesses (MTs) of the rectus abdominis (RA), external oblique, internal oblique (IO), and transverse abdominis were determined with an ultrasound apparatus. No significant changes for any measured variables were found in CON. In both training groups, the trunk segment LBM was significantly increased through the intervention. While MT for IO significantly increased in the two training groups, significant increases in MT for RA were found in only WP. For collegiate soccer players, the core training programs adopted here can be effective in increasing trunk segment LBM, but the effectiveness on the trunk flexor muscularity differs between the two training modalities.
\end{abstract}

\section{Introduction}

It is known that weakness in core stability or strength leads to low back pain, and core training can improve core strength and stability in patients with low back pain and athletes [11]. Strengthening the trunk can be a potential factor for enhancing torque development by limbs $[2,16]$. Trunk flexor muscles, consisting of the rectus abdominis, and external and internal oblique, contribute to the stability of the body in relation to trunk rotation, trunk and lateral flexion [3, 13]. Many different core training modalities such as body mass-based exercises $[5,17,20]$ and commercial devices such as the Swiss ball and wheeled roller [6, 7] have been developed to improve core strength and stability [11].
For athletes, available information concerning the effect of core training on trunk musculature is scarce. Among the limited findings, Hides et al. [12] have shown that abdominal drawing-in training increased the cross-sectional area of the multifidus in Australian football players with or without low back pain. On the other hand, Hoshikawa et al. [15], who applied core training consisting of body mass-based prone, supine, lateral plank, and push-up exercises to junior soccer players, failed to find significant changes in the muscularity of the rectus and lateral abdominal muscles. Hibbs et al. [11] maintain that muscle activation of $>60 \%$ of maximal voluntary contraction (MVC) is required to increase muscle size and strength. Combining the earlier findings with those that the activ- 
ity levels of the trunk flexor muscles during body mass-based core exercises have been shown to be $<40 \%$ of MVC $[5,17]$, the null findings of Hoshikawa et al. [15] may be due to low muscle activation during the adopted exercises.

Escamilla et al. [6] demonstrated that the use of wheel device induced higher muscular activities in the rectus abdominis and external/internal obliques than traditional body mass-based exercises such as the sit-up and crunch. From their findings, core exercises with wheeled devices produced muscular activities corresponding to $80 \%$ of MVC. This finding suggests that core training with wheeled devices would elicit greater hypertrophy in trunk flexor muscles, as compared to body mass-based core training. On the other hand, Mitchell et al. [19] have demonstrated that low-intensity training to failure resulted in similar hypertrophy in knee extensors as did training with a heavy load to failure. This finding tempts us to assume that body mass-based core exercises would also be a modality for increasing the muscularity of trunk muscles when they are continued to failure in each exercise session. To the best of our knowledge, however, no studies have examined whether core training with wheeled devices can be a modality for increasing trunk flexor musculature in athletes, and how its effectiveness on the muscularity differs from that induced by body mass-based core training.

The present study aimed to elucidate the effects of two core training programs on trunk flexor muscularity in athletes. To this end, we set up two programs for male soccer players: core training with wheeled platforms and core training consisting of body massbased exercises. We hypothesized that the two training programs would equally increase trunk flexor musculature.

\section{Methods}

\section{Subjects}

Twenty-eight collegiate male soccer players (19.9 $\pm 1.1 \mathrm{yrs}$, $171.1 \pm 5.8 \mathrm{~cm}, 65.3 \pm 6.4 \mathrm{~kg}$ ) were randomly assigned to three groups: Core exercises using wheeled platforms (WP, $N=10$ ), body mass-based core exercises (BME, $N=8)$, and a control group $(N=10$, $\mathrm{CON})$. This study constituted a randomized control trial. Based on the measured variables as hereinafter described, we allocated the participants to three groups so that no significant group-related differences in the corresponding variables were found. They were free of cardiovascular, metabolic, and immunologic disorders and/ or orthopedic abnormalities, and were not using any medications that affected their muscle functions. All participants had played soccer for over six years. Participants conducted soccer-specific training programs for at least $2 \mathrm{~h} /$ day on 6 days/wk. This investigation was conducted according to both the ethical standards of the Journal [10] and the Declaration of Helsinki, and was approved by the local University Ethics Committee for human experimentation. Prior to the experiment, all participants were informed of the experimental procedures of this study and possible risks of the measurements beforehand. Written informed consent was obtained from each subject.

\section{Experimental procedure}

All groups conducted soccer training according to nearly the same schedule, which consisted of ball possessions, small-sided games on the weekday ( $<2 \mathrm{~h} /$ day), and one friendly or official game on the weekend. Both training groups conducted the following program twice a week for 10 weeks in season. Each training program was altered every two weeks to increase training load. Training duration per session was approximately $50 \mathrm{~min}$. Both training groups also performed plyometric training on a different day during the same period ( 2 times/week, 10 weeks).

The BME group performed four core stabilization exercises consisting of prone, supine, lateral planks, and push-ups as described in Hoshikawa et al. [15]. Each exercise was performed to failure every set (2-3 sets/session) [19] ( $\triangleright$ Table 1 ), because muscle activations of the rectus abdominis during the adopted exercises have been shown to be $<40 \% \operatorname{MVC}[5,17,20]$.

Prone plank In a prone position on the floor with the elbow angle at $90 \mathrm{deg}$ and forearms placed underneath the chest, with the pelvis raised off the floor and their body mass distributed on the forearms and toes, the participants were asked to keep the body, including the back of the head, hips and heels, in a straight line.

Supine plank In a supine position on the floor with the elbow angle at $90 \mathrm{deg}$ and the forearms placed underneath the back, and with pelvis raised off the floor and their body mass distributed on the forearms and heels, the participants were instructed to hold the position.

Lateral plank While lying on the right or left side on the floor with the supporting elbow bent at $90 \mathrm{deg}$ and positioned directly under the shoulder, and with the pelvis raised off the floor and their body mass distributed on the forearms and the right or left side of the foot, the participants were asked to lift their hips and to maintain a straight line from head to toe.

Push-up Participants took a position in which their body mass was supported by both knees straight and both hands. They performed a push-up exercise at a tempo of every $4 \mathrm{~s}$.

The WP group performed 8-14 exercises each session with nylon wheeled platforms (PD-403-2N, $416 \mathrm{~mm} \times 275 \mathrm{~mm} \times 80 \mathrm{~mm}$, $1.3 \mathrm{~kg}$, Nansin), as presented in $>$ Fig. 1a. The exercises basically started from the following postures: 1) push-up position, feet on the wheeled platform (trunk, hips, knees, and elbows in full extension, shoulders flexed at $90 \mathrm{deg}$, and hands on the floor shoulderwidth apart), and 2) quadruped position, hands on the wheeled platform (knees, hips, and shoulders flexed approximately $90 \mathrm{deg}$, and elbows and trunk in full extension), and 3) forward-bending position, hands on the wheeled platform with knees extended. Participants were instructed to keep their trunk parallel to the floor, and not to tilt their knees forward as far as possible when their hips were flexed during each exercise ( $\vee$ Fig. 1b). The training program is presented in $>$ Table 2.

\section{Outcome measures}

To determine the effect of core training on trunk flexor musculature, trunk segment lean body mass (LBM) and muscle thicknesses (MTs) of the trunk flexor muscles were assessed before (Pre) and after (Post) the intervention. 
Trunk segment lean body mass (LBM) was measured using a whole-body dual-energy X-ray absorptiometry (DXA) scanner (Hologic Delphi A-QDR, USA). Participants lay supine on a bed with arms and legs straight. The room temperature was usually kept at $22^{\circ} \mathrm{C}$. The participants restrained from alcohol intake during $24 \mathrm{~h}$ and from taking a meal during the $2 \mathrm{~h}$ prior to the measurement. DXA-derived whole body and segmental LBM have good accuracy and reliability in team sport athletes [4].
Muscle thicknesses (MTs) of the rectus abdominis (RA), external oblique (EO), internal oblique (IO), and transverse abdominis (TrA) were measured using an ultrasound apparatus (Prosound Alpha 6, Hitach Aloka Medical, Japan) with a linear probe (7.27 MHz). The right side of the body was measured for all participants. During the measurements, the participants remained in a standing position with legs and arms straight and the muscle relaxed. The scanning head with a water-soluble transmission gel that

- Table 1 Body mass-based core training.

\begin{tabular}{|c|c|c|c|c|c|c|c|c|c|c|}
\hline & \multicolumn{2}{|c|}{ Week 1-2 } & \multicolumn{2}{|c|}{ Week 3-4 } & \multicolumn{2}{|c|}{ Week 5-6 } & \multicolumn{2}{|c|}{ Week 7-8 } & \multicolumn{2}{|c|}{ Week 9-10 } \\
\hline & Set & Time & Set & Time & Set & Time & Set & Time & Set & Time \\
\hline \multicolumn{11}{|l|}{ Prone plank } \\
\hline with both feet and elbows on floor & $2-3$ & $\mathrm{AO}$ & 3 & $\mathrm{AO}$ & & & & & & \\
\hline with one leg and arm raised & & & & & 2 & $\mathrm{AO}$ & 3 & $\mathrm{AO}$ & 3 & $\mathrm{AO}$ \\
\hline \multicolumn{11}{|l|}{ Supine plank } \\
\hline with both heels and elbows on floor & $2-3$ & $\mathrm{AO}$ & 3 & $\mathrm{AO}$ & & & & & & \\
\hline with one leg raised & & & & & 2 & $\mathrm{AO}$ & 3 & $\mathrm{AO}$ & 3 & $\mathrm{AO}$ \\
\hline \multicolumn{11}{|l|}{ Lateral plank } \\
\hline in straight line from head to feet & $2-3$ & $\mathrm{AO}$ & 3 & $\mathrm{AO}$ & & & & & & \\
\hline with one leg raised & & & & & 2 & $\mathrm{AO}$ & 3 & $\mathrm{AO}$ & 3 & $\mathrm{AO}$ \\
\hline \multicolumn{11}{|l|}{ Push-up } \\
\hline with both feet and hands on floor & $2-3$ & $\mathrm{AO}$ & 3 & $\mathrm{AO}$ & & & & & & \\
\hline with one leg raised & & & & & 2 & $\mathrm{AO}$ & 3 & $\mathrm{AO}$ & 3 & $\mathrm{AO}$ \\
\hline
\end{tabular}

a Wheeled platforms b used in this study

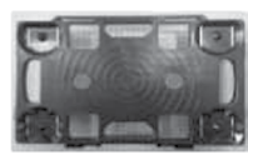

Correct posture during core training with wheeled platforms
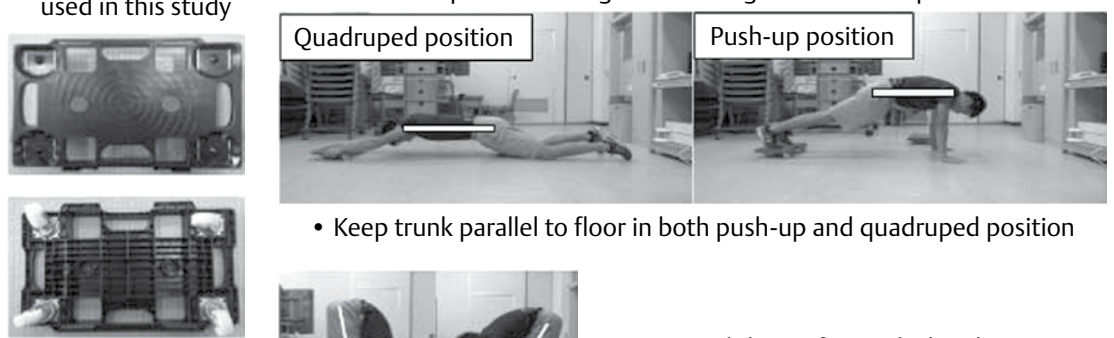

- Keep trunk parallel to floor in both push-up and quadruped position

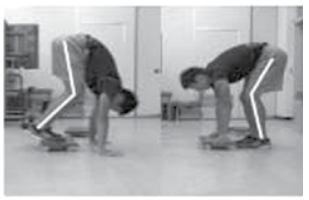

- Do not tilt knees forward when hips are flexed

- Roll out while kneeling (left) and standing (right) on floor

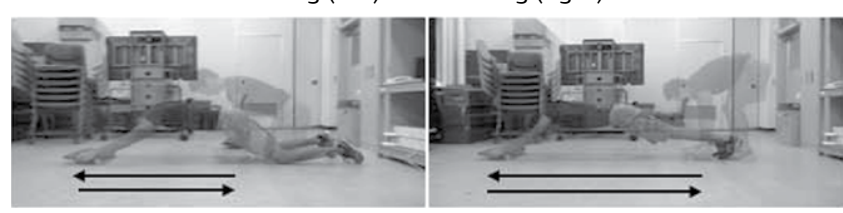

- V-shape roll-out with both hands on floor

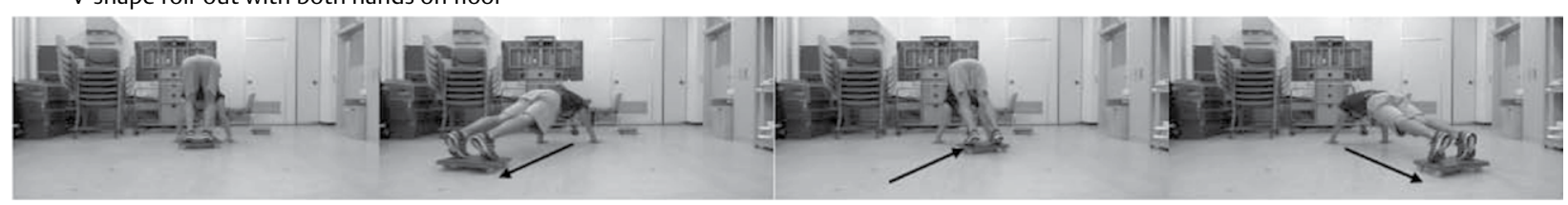

- Roll out with both hands on the floor

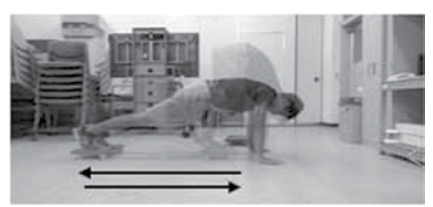


- V-shape roll-out while kneeling (upper) and standing (bottom) on floor

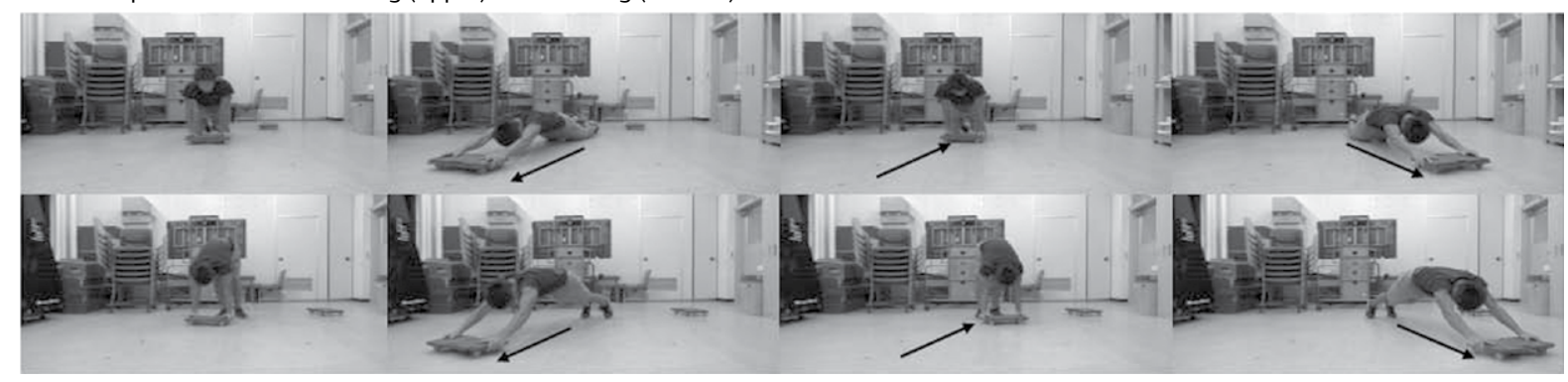

- Circle (reverse circle) while kneeling (upper) and standing (bottom) on floor

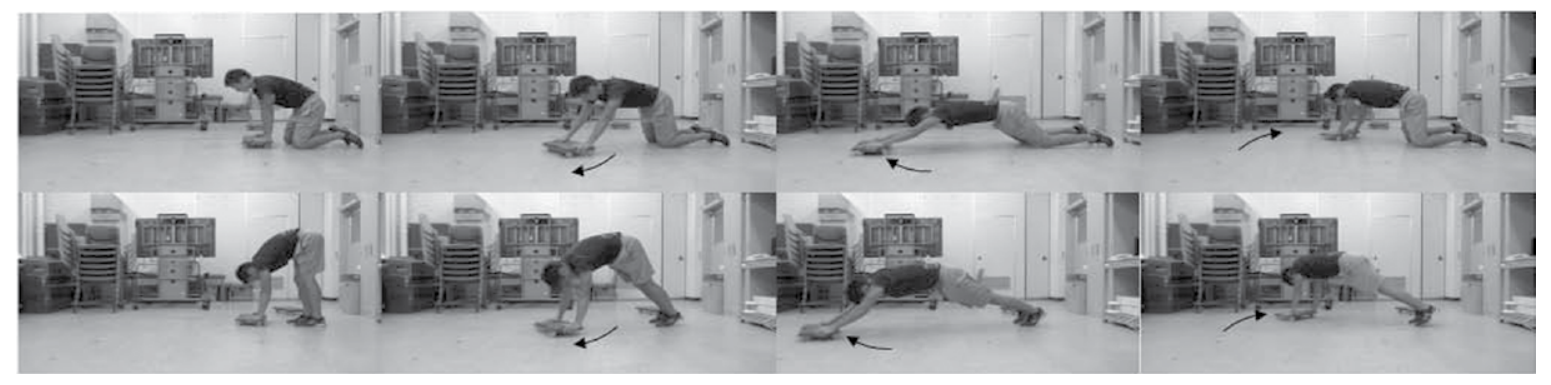

- Circle (reverse circle*) with both hands on floor

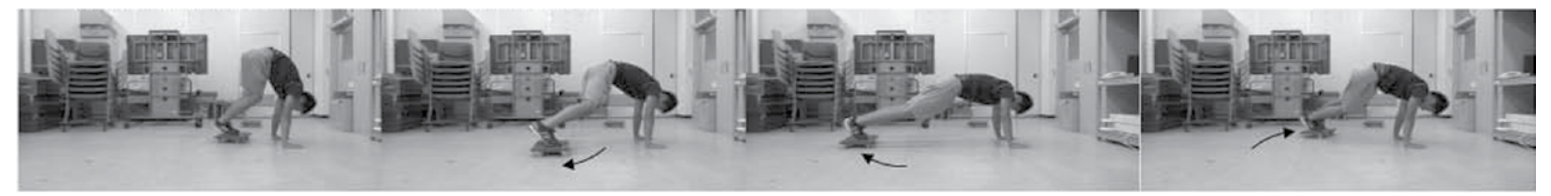

In the reverse circle exercise, the wheeled platform is moved clockwise, then counterclockwise.

- Wiper while kneeling (upper) and standing (bottom) on floor

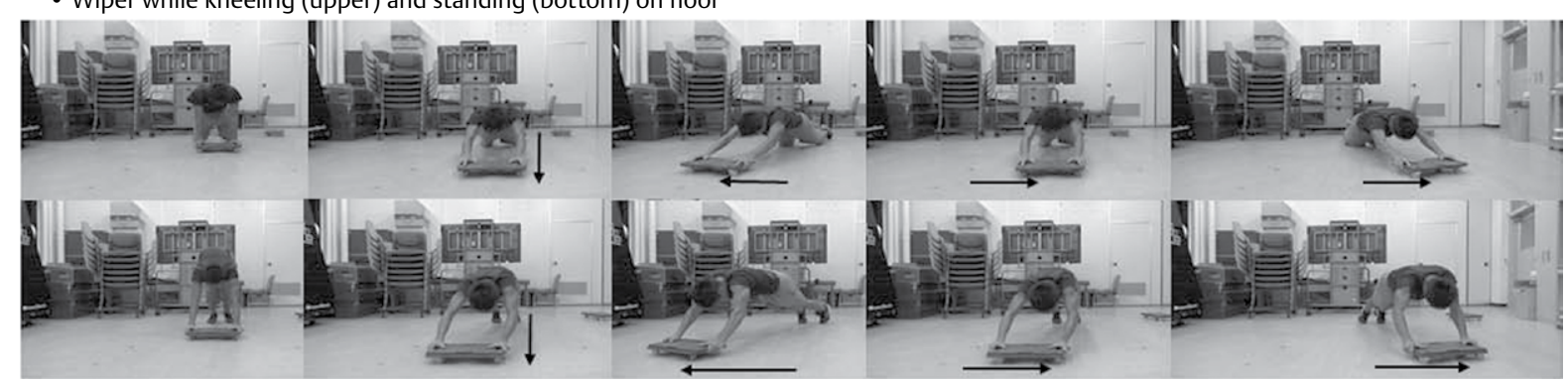

- Wiper with both hands on floor

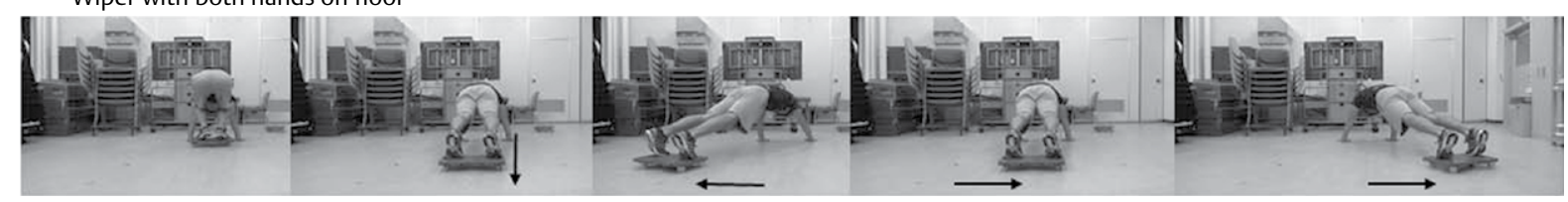

- Roll out with both hands and feet on wheeled platforms, fixed feet (right) and fixed hands (left)
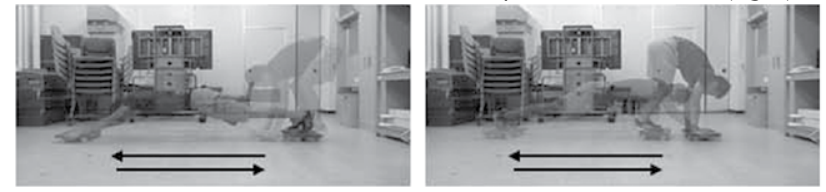

- A-shape with wheeled platforms

- Roll out/in with both hands and feet on wheeled platforms

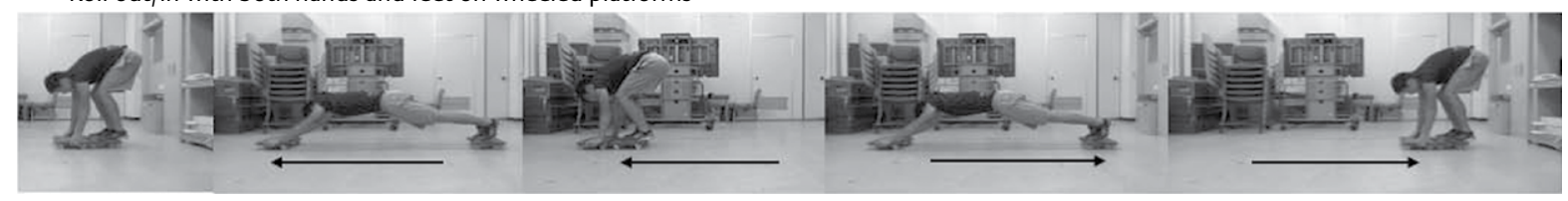

Fig. 1 Continued. 
- Worm (upper) and reverse worm (bottom) with wheeled platforms

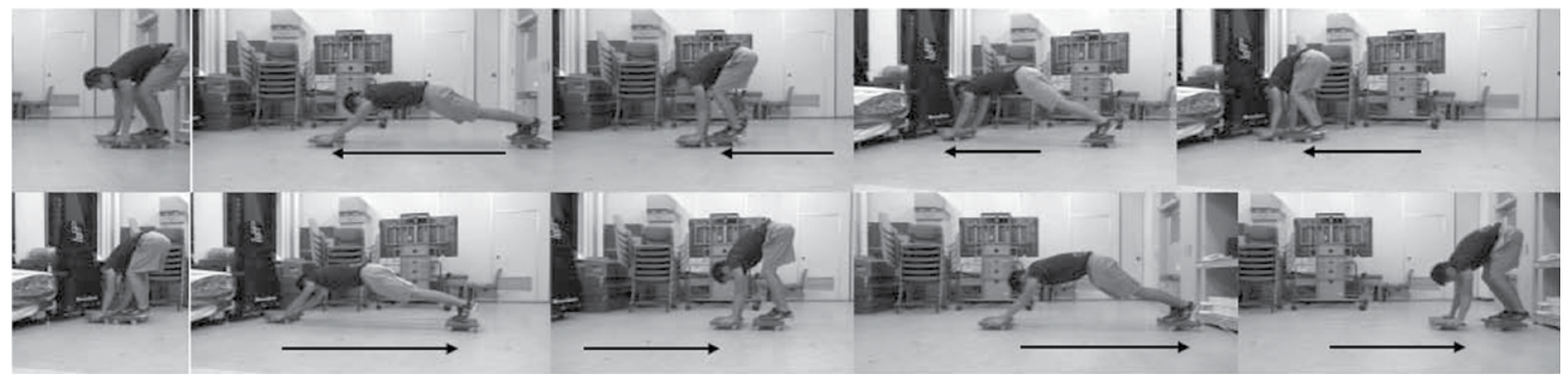

- Rotation with wheeled platforms

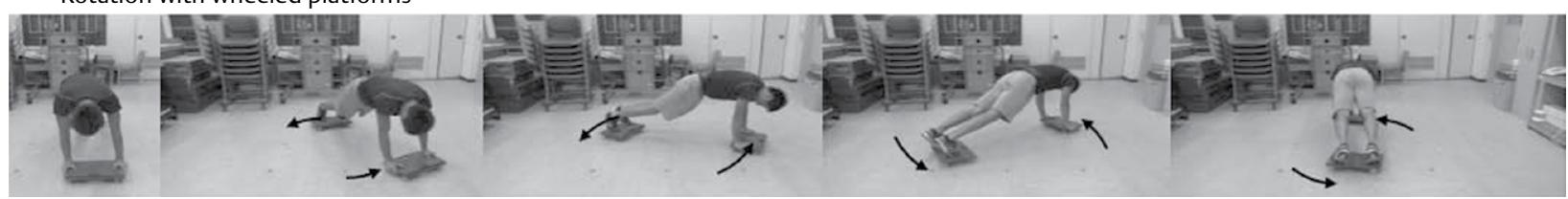

-Wiper \& A-shape with wheeled platforms

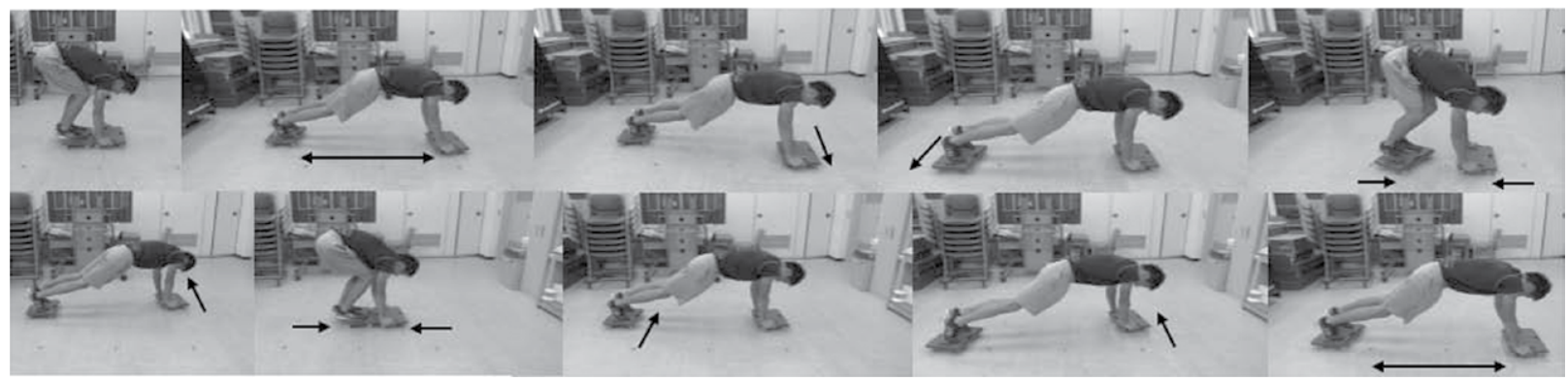

Fig. 1 Continued.

provided acoustic contact without depression of the skin surface was placed perpendicularly to the tissue interface. Distortion of tissues due to excess compression was eliminated, ensuring that no movement of tissues occurred in an image. MT for RA was measured at three sites: with the muscle belly positioned under the 2 nd tendinous intersection ( $\left.R A_{U P P E R}\right)$, the 3rd tendinous intersection $\left(R A_{M I D}\right)$, and the 4th tendinous intersection $\left(R A_{L O W E R}\right)$, respectively. At each of the measured sites, the MTs for RA were defined as the distances between the fat-muscle tissue interface and the muscle-abdominal cavity boundary. The MTs of EO, IO, and TrA were determined as distances between the fat-tissue interface and the EO-IO boundary, between the boundary and IO-TrA interface, and between the boundary and $\operatorname{TrA}$-abdominal cavity boundary, respectively. All images were analyzed by using image analysis software (Image J ver.1.47, NIH, USA). To confirm the repeatability of the MT measurements, an examiner measured the corresponding sites twice at an interval of 2 days. The intraclass correlation coefficients for the MT measurements were $\geq 0.87$. The measurement error between the $1 \mathrm{st}$ and $2 \mathrm{nd}$ measurements was $0.8 \pm 0.2 \mathrm{~mm}$, and the coefficient of variance was $5.3 \pm 4.4 \%$.

\section{Statistical analysis}

Descriptive data are means and SDs. One-way analysis of variance (ANOVA) was used to examine the group-related differences in all measured variables at baseline. To examine the time-induced changes in trunk segment LBM and MTs of the trunk flexors, twoway ANOVA with repeated measures (group by time) was used to examine the main effects and interaction. When appropriate, a simple main effect test was used to test the significance of the changes in the measured variables. Cohen's $d$ was used to examine the effect size (ES) of the difference between Pre and Post in the measured variables in each group, and its magnitude was interpreted in accordance with the recommendations of Hopkins [14], defining < $0.2,0.2-0.6,0.6-1.2,1.2-2.0,2.0-4.0$, and $>4.0$ as trivial, small, moderate, large, very large, and nearly perfect, respectively. All statistical analyses were performed using statistical software (SPSS ver. 23, IBM, Japan). The level of significance was set at $\mathrm{p}<0.05$.

\section{Results}

Descriptive data are presented in $>$ Table 3. At baseline, no significant group-related differences in the measured variables were found. ANOVAs revealed that there were significant main effects of time in trunk segment LBM $(F=5.69, p=0.025)$ and MT for IO $(F=6.90, p=0.015)$ without significant interaction ( $\vee$ Table 3$)$. The ESs of trunk segment LBM were $0.10-0.18$ across groups, corresponding to trivial, but those of MT for 10 were 0.80 in WP, and 
- Table 2 Core training with wheeled platforms.

\begin{tabular}{|c|c|c|c|c|c|c|c|c|c|c|}
\hline & \multicolumn{2}{|c|}{ Week 1-2 } & \multicolumn{2}{|c|}{ Week 3-4 } & \multicolumn{2}{|c|}{ Week 5-6 } & \multicolumn{2}{|c|}{ Week 7-8 } & \multicolumn{2}{|c|}{ Week 9-10 } \\
\hline & Set & Time & Set & Time & Set & Time & Set & Time & Set & Time \\
\hline Roll out while kneeling on the floor & 1 & 8 & 1 & $8-10$ & 1 & 10 & 1 & $5(\mathrm{ST})$ & 1 & $5(\mathrm{ST})$ \\
\hline Roll out with both hands on the floor & & 8 & & $8-10$ & & 10 & & 10 & & 10 \\
\hline V-shape roll-out while kneeling on the floor & & 8 & & $8-10$ & & 10 & & $6(\mathrm{ST})$ & & $6(\mathrm{ST})$ \\
\hline V-shape roll-out with both hands on the floor & & 8 & & $8-10$ & & 10 & & 10 & & 10 \\
\hline Circle while kneeling on the floor & & $3 \#$ & & - & & - & & 4(ST)\# & & - \\
\hline Circle with both hands on the floor & & $3 \#$ & & - & & - & & $4 \#$ & & - \\
\hline Reverse circle while kneeling on the floor & & $3 \#$ & & - & & - & & 3(ST)\# & & - \\
\hline Reverse circle with both hands on the floor & & $2 \#$ & & - & & - & & 3\# & & - \\
\hline Wiper while kneeling on the floor & & 3 & & - & & - & & $1(\mathrm{ST})$ & & - \\
\hline Wiper with both hands on the floor & & 3 & & - & & - & & 4 & & - \\
\hline Roll out on wheeled platforms (fixed feet) & & 3 & & - & & - & & 3 & & - \\
\hline Roll out on wheeled platforms (fixed hands) & & 3 & & - & & - & & 3 & & - \\
\hline Roll out/in on wheeled platforms & & 3 & & - & & - & & 3 & & - \\
\hline A-shape with wheeled platforms & & $10 \mathrm{~s}$ & & - & & - & & $10 \mathrm{~s}$ & & - \\
\hline Worm with wheeled platforms & & - & & 3 & & 3 & & - & 3 & 3 \\
\hline Reverse worm with wheeled platforms & & - & & 3 & & 3 & & - & 3 & 3 \\
\hline Rotation with wheeled platforms & & - & & $2(180)$ & & $2(180)$ & & - & $2(360)$ & $2(360)$ \\
\hline Wiper \& A-shape with wheeled platforms & & - & & 2 & & 2 & & - & 2 & 2 \\
\hline
\end{tabular}

- Table 3 Descriptive data for the measured variables at Pre and Post for all groups.

\begin{tabular}{|c|c|c|c|c|c|c|}
\hline & \multicolumn{2}{|c|}{ WP } & \multicolumn{2}{|c|}{ STB } & \multicolumn{2}{|c|}{ CON } \\
\hline & Pre & Post & Pre & Post & Pre & Post \\
\hline Body height, cm & $170.2(6.6)$ & $170.6(6.5)$ & $170.1(6.2)$ & $170.2(6.1)$ & $172.9(4.7)$ & $173.3(4.5)$ \\
\hline Body mass, kg & $64.1(8.0)$ & $65.1(8.0)^{*}$ & $65.8(5.9)$ & $66.3(5.5)^{*}$ & $66.2(5.3)$ & $66.0(5.8)$ \\
\hline \%FM, \% & $11.7(2.7)$ & $11.9(2.9)$ & $11.8(2.1)$ & $11.9(2.2)$ & $11.5(2.6)$ & $10.8(2.0)$ \\
\hline Fat mass, kg & $7.5(2.3)$ & $7.8(2.6)$ & $7.8(1.7)$ & $7.9(1.6)$ & $7.6(2.1)$ & $7.2(1.8)$ \\
\hline \multicolumn{7}{|c|}{ Lean body mass, $\mathrm{kg}$} \\
\hline Whole body & $54.0(6.2)$ & $54.7(6.1)^{*}$ & $55.5(5.0)$ & $55.9(4.9)^{*}$ & $56.0(4.0)$ & $56.2(4.3)$ \\
\hline Trunk & $26.0(3.1)$ & $26.6(3.2)^{*}$ & $26.8(2.3)$ & $27.2(2.4)^{*}$ & $27.0(2.2)$ & $27.2(2.3)$ \\
\hline \multicolumn{7}{|c|}{ Muscle thickness, mm } \\
\hline $\mathrm{RA}_{\text {UPPER }}$ & $14.0(2.2)$ & $16.1(2.1)^{*}$ & $15.0(2.5)$ & $14.9(2.9)$ & $15.0(2.6)$ & $15.4(3.1)$ \\
\hline $\mathbf{R A}_{\text {MID }}$ & $15.2(2.4)$ & $17.1(2.4)^{*}$ & $17.1(3.5)$ & $16.4(2.6)$ & $16.3(2.6)$ & $16.4(2.7)$ \\
\hline RA $_{\text {LOWER }}$ & $14.9(1.7)$ & $17.6(2.1)^{*}$ & $16.7(2.3)$ & $16.3(2.9)$ & $16.0(2.2)$ & $16.3(2.5)$ \\
\hline EO & $9.3(1.6)$ & $10.1(1.2)$ & $9.3(1.4)$ & $10.0(1.2)$ & $10.3(1.8)$ & $10.2(1.2)$ \\
\hline 10 & $15.0(2.0)$ & $16.6(1.6)^{*}$ & $16.7(2.9)$ & $17.6(2.4)^{*}$ & $16.6(1.8)$ & $17.2(2.7)$ \\
\hline TrA & $4.8(1.3)$ & $5.0(1.5)$ & $5.6(1.7)$ & $5.3(1.4)$ & $4.8(1.1)$ & $4.8(1.2)$ \\
\hline
\end{tabular}

Values are expressed as means (SDs). FM, fat mass; RA, rectus abdominis; EO, external oblique; IO, internal oblique; TrA, transverse abdominis

0.25-0.33 in other groups, corresponding to moderate and small, respectively. MTs for RA had significant interaction ( $F=14.60$, $p<0.001)$. Regardless of the measured sites, MTs for RA significantly increased in WP with moderate to large ES (0.97-1.62), but not in BME and CON with trivial to small effect size $(<0.20)$. MTs for EO and TrA did not change significantly through the intervention. The ESs of MT for EO were 0.49-0.52 in WP and BME and -0.06 in CON, corresponding to small and trivial, respectively. The ESs of MT for TrA were trivial $(<0.20)$ across all groups.

\section{Discussion}

The main finding obtained here was that, for male soccer players, the core training with wheeled platforms increased MTs for RA and $\mathrm{IO}$, but a corresponding gain with body mass-based core training was limited to the MT for IO. This indicates that although core training programs with either body mass-based exercises or wheeled platforms can be modalities to increase the trunk flexor muscularity in athletes, their effectiveness is muscle-specific.

The trunk segmental LBM was significantly increased through the interventions in all groups with trivial effect size. The mean val- 
ues of the relative changes in the corresponding variables were 1.5$2.1 \%$ in both groups, and $0.8 \%$ in CON. As a seasonal variation, trunk segmental LBMs for professional male soccer players have been shown to change by $0.4 \%$ [18], being similar to the observed relative change in CON. Compared to these values, the relative gains in the two training groups are higher, indicating that the observed changes are training effect, not seasonal variation.

Significant gain in MT for RA was limited to WP. It is known that the occurrence of training-induced hypertrophic change in a muscle depends on its activation level during the exercise adopted [24]. Therefore, the observed muscle-specific change in thicknesses may be assumed to be due to the differences in the activation levels among the muscles involved in the prescribed exercises. In an earlier study [6], the muscle activation of the abdominal muscles (the upper and lower rectus abdominus muscles, internal and external oblique muscles) during the exercises with a wheeled roller reaches up to $80-90 \%$ of MVC. In this protocol, the activation levels of EO during prone plank with one leg and arm raised, as well as lateral plank exercises are $>80 \%$ of MVC [20]. In previous studies $[5,17,20]$, the activation level of RA during the core exercises adopted in this study has been shown to be less than $40 \%$ of MVC. These findings might explain the observed differences between the two training protocols adopted here in the change in MT for RA, although we did not measure the activation levels of the trunk flexor muscles during the prescribed exercises.

The observed difference between the two training groups in the hypertrophic change differed from our hypothesis. In this study, we designed training protocols in which the participants were required to perform the adopted core exercises until failure per set. This is based on the earlier findings that a training program with a low load produced a similar hypertrophic change as that with a high load when exercises were conducted to failure per set [19]. Thus, at the start of this study, we expected that the two training protocols would produce similar hypertrophic change in trunk flexor muscles. However, the current result denies this assumption. A possible explanation for this discrepancy is the initial levels of MTs for the participants in this study. Namely, the MTs of the abdominal muscles in this study (RA, 15-17 mm; EO, 9-10 mm; IO, 15-17 mm) were thicker than those previously reported for active males [23] and patients with low back pain [21] (RA, 8-15 mm; EO, 5-8 mm; IO, $5-14 \mathrm{~mm})$. Muscle size is related to force-generating capacity [1,9]. Muscle activation level during body mass-based exercises is negatively related to the force-generating capacity $[8,22]$. Considering these aspects, it seems that the room for increasing trunk flexor muscularity in the participants would be small, and consequently BME compared to WP might have produced less hypertrophic changes in abdominal muscles in relation to the aforementioned differences in the muscular activity levels during the prescribed exercises.

We should state some limitations in this study. First, the training-induced changes in the muscle thicknesses of the trunk flexors differed between the WP and BME groups, whereas the change in trunk LBM was almost equivalent for the two training groups. Yang et al. [25] have demonstrated that back and side bridge exercise training increased not only lateral abdominis MT but also the mul- tifidus MT in healthy young men. When these findings are combined with the current findings, their training may be assumed to have elicited muscles such as the multifidus muscles and the back extensors, unlike the subject muscles measured here. Unfortunately, we did not have the relevant data, and further investigation is needed on this point. Second, the training-induced changes in the trunk flexors might be due to conducting plyometric training in both training groups, because the CON group did not perform them. If so, the magnitude of increase of the muscle thicknesses would be same in both groups. However, the changes in the rectus abdominis differed between WP and BME. Therefore, the effect of the plyometric training on the trunk flexors may be less in this study. Finally, the sample size in this study was small because the subjects were recruited in a team. This may result in Type I or II errors as a potential bias. Hence we have calculated effect size according to the earlier study [14]. In the current findings, ES for the trunk flexors indicated small to large for WP, small for BME, and trivial to small for CON. These results indicate that the two training modalities may be superior to soccer-specific training alone for producing hypertrophic changes in the trunk flexor muscles.

In terms of practical application, the present results indicate that, for soccer players, core training with wheeled platforms may be superior to body mass-based core training for developing trunk flexor muscle size, in particular the rectus abdominis, compared to core training with body mass-based exercises. Training-induced muscle hypertrophy can lead to enhanced force-generating capacity. Strengthening the trunk flexor muscles may contribute to increased torque generated by the limbs $[2,16]$. Therefore, it may be assumed that the core training program with wheeled platforms can be feasible as modalities for improving trunk flexor muscles in soccer players.

\section{Conclusion}

For increasing muscularity of the rectus and lateral abdominis, core training with wheeled platforms is superior to that consisting of body mass-based exercises. The present findings suggest that the selection of core exercises requires careful consideration because of the possible occurrence of muscle-specific hypertrophic change within the trunk flexor muscles through the exercise protocols adopted.

\section{Acknowledgements}

This work is supported by a Grant-in-Aid for Scientific Research (C) (26350748)

\section{Conflict of Interest}

The authors declare that they have no conflict of interest. 


\section{References}

[1] Akagi R, Takai Y, Ohta M, Kanehisa H, Kawakami Y, Fukunaga T. Muscle volume compared to cross-sectional area is more appropriate for evaluating muscle strength in young and elderly individuals. Age Ageing 2009; 38: 564-569

[2] Behm DG, Anderson KG. The role of instability with resistance training. J Strength Cond Res 2006; 20: 716-722

[3] Bergmark A. Stability of the lumbar spine. A study in mechanical engineering. Acta Orthop Scand Suppl 1989; 230: 1-54

[4] Bilsborough JC, Greenway K, Opar D, Livingstone S, Cordy J, Coutts AJ. The accuracy and precision of DXA for assessing body composition in team sport athletes. J Sports Sci 2014; 32: 1821-1828

[5] Byrne JM, Bishop NS, Caines AM, Crane KA, Feaver AM, Pearcey GE. Effect of using a suspension training system on muscle activation during the performance of a front plank exercise. J Strength Cond Res 2014; 28: 3049-3055

[6] Escamilla RF, Babb E, DeWitt R, Jew P, Kelleher P, Burnham T, Busch J, D'Anna K, Mowbray R, Imamura RT. Electromyographic analysis of traditional and nontraditional abdominal exercises: Implications for rehabilitation and training. Phys Ther 2006; 86: 656-671

[7] Escamilla RF, Lewis C, Bell D, Bramblet G, Daffron J, Lambert S, Pecson A, Imamura R, Paulos L, Andrews JR. Core muscle activation during Swiss ball and traditional abdominal exercises. J Orthop Sports Phys Ther 2010; 40: 265-276

[8] Fujita E, Kanehisa H, Yoshitake Y, Fukunaga T, Nishizono H. Association between knee extensor strength and EMG activities during squat movement. Med Sci Sports Exerc 2011; 43: 2328-2334

[9] Fukunaga T, Miyatani M, Tachi M, Kouzaki M, Kawakami Y, Kanehisa H. Muscle volume is a major determinant of joint torque in humans. Acta Physiol Scand 2001; 172: 249-255

[10] Harriss D], Atkinson G. Ethical standards in sport and exercise science research: 2016 update. Int J Sports Med 2015; 36: 1121-1124

[11] Hibbs AE, Thompson KG, French D, Wrigley A, Spears I. Optimizing performance by improving core stability and core strength. Sports Med 2008; 38: 995-1008

[12] Hides JA, Stanton WR, Mendis MD, Gildea J, Sexton MJ. Effect of motor control training on muscle size and football games missed from injury. Med Sci Sports Exerc 2012; 44: 1141-1149

[13] Hodges PW. Is there a role for transversus abdominis in lumbo-pelvic stability? Man Ther 1999; 4: 74-86
[14] Hopkins WG. Linear models and effect magnitudes for research, clinical and practical applications. Sportscience 2010; 14

[15] Hoshikawa Y, lida T, Muramatsu M, li N, Nakajima Y, Chumank K, Kanehisa $\mathrm{H}$. Effects of stabilization training on trunk muscularity and physical performances in youth soccer players. J Strength Cond Res 2013; 27: 3142-3149

[16] Kibler WB, Press ], Sciascia A. The role of core stability in athletic function. Sports Med 2006; 36: 189-198

[17] Maeo S, Takahashi T, Takai Y, Kanehisa H. Trunk muscle activities during abdominal bracing: comparison among muscles and exercises. J Sports Sci Med 2013; 12: 467-474

[18] Milanese C, Cavedon V, Corradini G, De Vita F, Zancanaro C. Seasonal DXA-measured body composition changes in professional male soccer players. J Sports Sci 2015; 33: 1219-1228

[19] Mitchell C], Churchward-Venne TA, West DW, Burd NA, Breen L, Baker SK, Phillips SM. Resistance exercise load does not determine training-mediated hypertrophic gains in young men. J Appl Physiol (1985) 2012; 113: 71-77

[20] Okubo Y, Kaneoka K, Imai A, Shiina I, Tatsumura M, Izumi S, Miyakawa S. Electromyographic analysis of transversus abdominis and lumbar multifidus using wire electrodes during lumbar stabilization exercises. J Orthop Sports Phys Ther 2010; 40: 743-750

[21] Shamsi M, Sarrafzadeh J, Jamshidi A, Zarabi V, Pourahmadi MR. The effect of core stability and general exercise on abdominal muscle thickness in non-specific chronic low back pain using ultrasound imaging. Physiother Theory Pract 2016; 32: 277-283

[22] Takai Y, Sawai S, Kanehisa H, Kawakami Y, Fukunaga T. Age and sex differences in the levels of muscular activities during daily physical actions. Int J Sport Health Sci 2008; 6: 169-181

[23] Tayashiki K, Maeo S, Usui S, Miyamoto N, Kanehisa H. Effect of abdominal bracing training on strength and power of trunk and lower limb muscles. Eur ] Appl Physiol 2016; 116: 1703-1713

[24] Wakahara T, Miyamoto N, Sugisaki N, Murata K, Kanehisa H, Kawakami Y, Fukunaga T, Yanai T. Association between regional differences in muscle activation in one session of resistance exercise and in muscle hypertrophy after resistance training. Eur J Appl Physiol 2012; 112: 1569-1576

[25] Yang HS, Lee YS, Jin SA. Effect of evidence-based trunk stability exercises on the thickness of the trunk muscles. J Phys Ther Sci 2015; 27: $473-475$ 